\section{A Review of a Presentation Technology: Prezi}

\author{
Brian E. Perron' and Alyson G. Stearns ${ }^{2}$
}

Social work researchers and educators need to effectively communicate information in a variety of settings. A successful presentation requires the content to be clear and engaging. Presentations are usually created with a software package (e.g., Microsoft PowerPoint), which enables users to construct slides for holding text, images, video, and animations to facilitate the delivery of information. In this review, we examine an innovative alternative to presentation software called Prezi (www.prezi.com).

\section{What is It?}

Prezi is an online presentation service provider that offers different types of accounts and options for creating and storing digital presentations. Traditional presentation software requires preparing a linear story line using a storyboard approach. Prezi, on the other hand, allows for both a linear and a free-flowing presentation of a story line. The user creates a presentation on a large blank workspace called the canvas, where all the elements of a presentation are visible. A story line is then created by arranging the elements on the canvas. Various tools are used to connect these elements in order to communicate the presenter's message. Like traditional presentation software, Prezi has the capability of integrating text, images, animation, audio, and video seamlessly into a single presentation.

\section{How Does It Work?}

An individual or organization chooses one of the subscription options on the Prezi website. Once a user account has been established, the subscription holder then logs in to the Prezi website and is provided with a canvas and tools for creating a presentation. The user is able to create new presentations, edit saved works, and view stored presentations directly on the website.

To create a presentation, users add topics and main ideas to the Prezi canvas. This canvas serves as a map for the entire presentation. As users add ideas to the canvas, they are able to zoom in and out, adding supplementary details and subtopics. Users can also insert images, shapes, documents, or videos anywhere among the presentation content, in addition to grouping ideas using frames. Paths are then specified between frames
Research on Social Work Practice $000(00)$ I-2

(C) The Author(s) 2010

Reprints and permission: sagepub.com/journalsPermissions.nav DOI: $10.1177 / 1049731510390700$ http://rsw.sagepub.com

@SAGE and individual elements to control the flow and order of the presentation. When a presentation is viewed, each topic is visited in the order specified by these paths.

Prezi provides an environment for creating highly customizable presentations. The user can select different fonts, colors, and canvas layouts and can incorporate different types of media. The elements can be easily rearranged anywhere on the canvas with the mouse, and further editing (e.g., size, orientation, and relative position) is done with an embedded editing tool. Users also have the option to publish their presentations online, where other subscribers may view and copy them.

Unlike many other presentation applications, Prezi's webbased interface allows users to access presentations on any computer with a high-speed Internet connection. Presentations can also be shared, allowing multiple people to collaborate on a single presentation. This is an important feature, given the growth of distance collaborations among social work researchers.

\section{Who is Using It?}

Prezi is relatively new, so the technology is still unfamiliar to researchers and educators, especially in the field of social work. However, it has tremendous promise for communicating information and ideas in both research and educational settings. In addition to the intuitive user interface, the collaborative features of Prezi are likely to aid in the adoption of this new technology.

\section{What Skills and Knowledge are Needed to Use This Technology?}

In our experience, Prezi presentations are relatively easy to create. Users of Prezi should be skilled at Internet navigation and file management. It would be advantageous to have a good

\footnotetext{
'University of Michigan, Ann Arbor, MI, USA

${ }^{2}$ University of Oklahoma, Norman, OK, USA

Corresponding Author:

Brian E. Perron, 1080 S. University Avenue, Ann Arbor, MI 48109, USA

Email: beperron@umich.edu
} 
understanding of design principles, as some of the features (e.g., animations) can be overused or used ineffectively, thus detracting from the presentation itself. However, this is a potential problem for users of any presentation software, not just Prezi. Furthermore, various challenges are encountered whenever new technologies are adopted. Such challenges can be easily addressed on the Prezi website, which contains tutorials and forums to assist with design and technical problems. In addition, Prezi technology support is also available online.

\section{What are the Technology Requirements?}

Prezi can be used with either a PC or Macintosh computer. Users need a high-speed Internet connection, an up-to-date supported web browser and operating system, a minimum of $1 \mathrm{~GB}$ computer memory, a scroll mouse or touchpad, and a recent version of the Adobe Flash Player program. Further details on computer requirements are available on the Prezi website.

\section{Why is It Significant?}

Prezi can be used as a tool for creating dynamic and informative presentations. Unlike many existing presentation tools, Prezi allows users to work on and access their presentations online, as well as on their local computer. Other presentation tools require content to fit within the boundaries of a slide, whereas Prezi allows users to prepare and present content of virtually any size. The presenter can focus on different elements by using the zooming and panning features. This can save a significant amount of time in preparing a presentation, and such capabilities can also help the audience view bigpicture concepts and specific details, which can greatly aid in comprehension and retention of information.

\section{What are the Downsides?}

In our experience, the Prezi website is intuitive and has several tutorials and options to help users get started with this technology. However, users who are not skilled at working with different file types and navigating websites may find Prezi confusing at first. At the time of writing this review, Prezi does not offer live technical support. In addition, some users may encounter problems loading Prezi if their system (i.e., hardware and software) is not up to date.
Prezi is a subscription-based service, and some features may not be affordable to all persons or organizations. The free account option that Prezi offers still requires that users have access to an updated computer with an Internet connection, which may not always be available to some participants. Finally, as is the case with almost any new technology, some users may be reluctant to adapt to the unstructured format of the Prezi canvas. However, in our experience, when the information to be presented is well-suited to this type of format, the response to it has been excellent.

\section{What are the Implications for Social Work Research?}

Prezi shows tremendous promise for enhancing the presentation of ideas in social work research and educational settings. This software rectifies design limitations of existing presentation software. Additionally, the ability to collaborate on the development of presentations can be of value to researchers working in remote areas. This feature is also important for educational purposes, particularly in promoting collaboration among students on joint projects. Overall, Prezi can be one of many important tools for enhancing the knowledge base of social work.

\section{Pricing Information}

Pricing varies by subscription plan, all of which are billed annually. At this time, a free account is available for users to create and store presentations with a small amount of storage space. However, individuals with an academic affiliation are entitled to a free upgrade to a fully functioning system with additional storage space. Users have the option to upgrade from the basic free account to a paid account at any time. Refer to www.prezi.com for current pricing information.

\section{Declaration of Conflicting Interests}

The author(s) declared no potential conflicts of interests with respect to the authorship and/or publication of this article.

\section{Funding}

The author(s) received no financial support for the research and/or authorship of this article. 